\title{
Novel Conformation of Single Ring GroEL-GroES Suitable for Encapsulation of an 86 kDa Assembly Intermediate as Visualized by Electron Cryomicroscopy
}

\author{
Dong-Hua Chen, ${ }^{*}$ Jiu-Li Song, ${ }^{* *}$ David T. Chuang, ${ }^{* *}$ Wah Chiu* and Steve Ludtke* \\ *National Center for Macromolecular Imaging, Verna and Marrs McLean Department of \\ Biochemistry and Molecular Biology, Baylor College of Medicine, Houston, TX 77030. \\ **Department of Biochemistry, University of Texas Southwestern Medical School, Dallas, TX \\ 75390.
}

Chaperonins GroEL/GroES promote proper folding and assembly of proteins[1]. Efficient folding of unfolded proteins was shown in the past to occur inside the cis cavity of GroEL encapsulated by GroES in the presence of Mg-ATP. The established size limit of the polypeptide that could be capped by GroES inside the cavity is believed to be $57 \mathrm{kDa}$ as shown by both in vivo and in vitro studies. The $86-\mathrm{kDa} \alpha \beta$ heterodimeric protein is an assembly intermediate in the formation of the mitochondrial branched-chain $\alpha$-ketoacid dehydrogenase (BCKD) catalytic machine. We have shown previously that GroEL/GroES are essential for promoting the conversion of a kinetically trapped heterodimer $(\alpha \beta)$ to the native heterotetrameric $\left(\alpha_{2} \beta_{2}\right)$ decarboxylase $(\mathrm{E} 1)$ component of the human BCKD complex[2]. Surprisingly, the 86-kDa heterodimer was found to be capped by GroES for both GroEL and its single ring mutant (SR1), as indicated by the protection of both the $\alpha$ and $\beta$ subunits from the protease digestion in the presence of nucleotides[2-3]. However, the encapsulation of this large native-like heterodimeric intermediate inside the chaperonin cis cavity by GroES has not been demonstrated at the structural level.

In the present study, we have used an ATPase-deficient single-ring GroEL mutant (SR398) as a model to visualize by Electron Cryomicroscopy the $86-\mathrm{kDa}$ heterodimer $(\alpha \beta)$ inside its single cavity encapsulated by GroES. Through an extensive set of Electron Cryomicroscopy experiments, combined with digestion protection experiments, and by using multiple-model refinement from single particle reconstruction package EMAN [4-5], we have identified a heretofore undiscovered expanded conformation of SR398-GroES-ATP besides the normal conformation that matches the published X-ray crystal structure of GroEL-GroES-(ADP) $)_{7}$ complex (referred to as crystal-structurelike conformation), and show conclusive evidence for the encapsulation of an 86-kDa heterodimer inside the cavity of SR398 associated with GroES, which significantly exceeds the proposed size limit of $57 \mathrm{kDa}$ established for unfolded protein inside the cis cavity of GroEL. The altered and novel conformation may have implications for chaperonins that exist in a single ring as well as for native GroEL.

References

[1] Fenton, W.A. and A.L. Horwich, Q Rev Biophys, 36 (2003) 229.

[2] Song, J.L., R.M. Wynn, and D.T. Chuang, J Biol Chem, 275 (2000) 22305.

[3] Song, J.L., et al., J Biol Chem, 278 (2003) 2515.

[4] Ludtke, S.J., P.R. Baldwin, and W. Chiu, J Struct Biol, 128 (1999) 82.

[5] Brink, J., et al., Structure (Camb), 12 (2004) 185. 


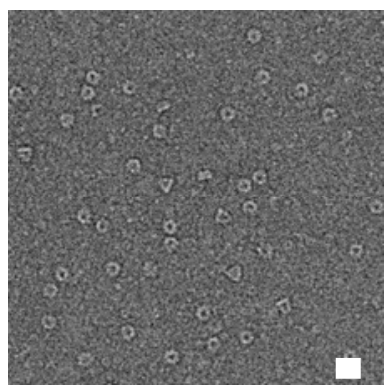

A

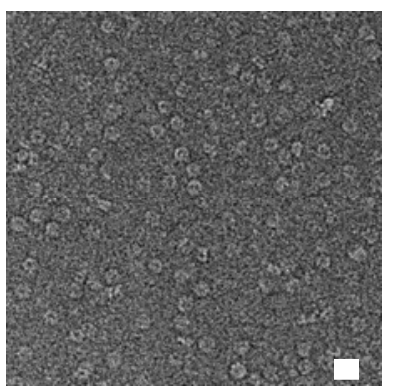

$\mathrm{B}$

Figure 1. Typical Cryo-images Collected on Gatan $4 \mathrm{k} \times 4 \mathrm{k}$ CCD in a JEOL2010F Electron Cryomicroscope (A) SR398-GroES-ATP complex. (B) SR398- $\alpha \beta$-GroES-ATP complex. The scale bar in both images is $200 \AA$.

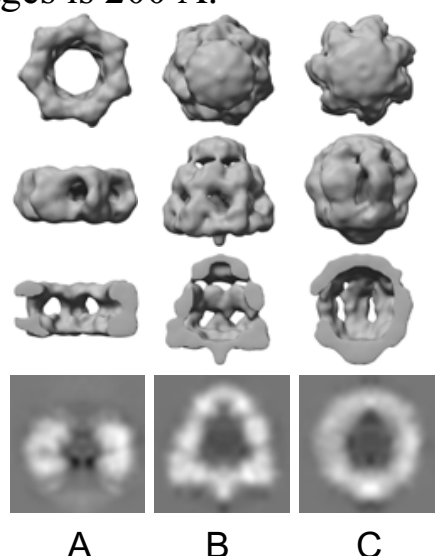

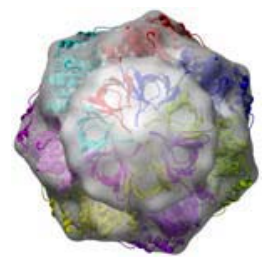

D

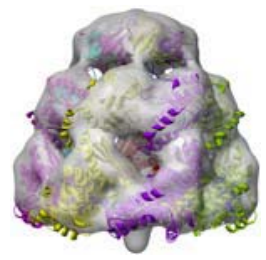

E

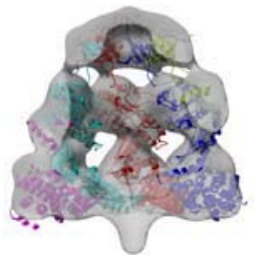

$\mathrm{F}$

Figure 2. Structures of three different conformations from heterogeneous SR398-GroES-ATP complex data at about $20 \AA$ (A-C) and X-ray crystallographic structure of GroEL-GroES-(ADP) 7 $(1 \mathrm{AON})$ with lower GroEL single ring cut away docked into Cryo-EM density map of crystalstructure-like conformation from the SR398-GroES-ATP Complex (D-F). (A) SR398 heptamer with no GroES. (B) Crystal-structure-like conformation. (C) Expanded conformation, representing a new conformation for chaperonin family. (D) Fitting for top view. (E) Fitting for side view. (F) Fitting for side view of the map with front half density cut away along the symmetry axis.

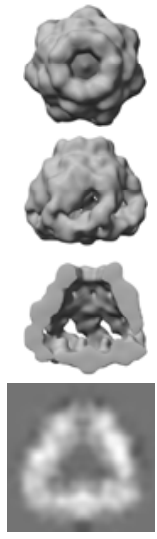

A
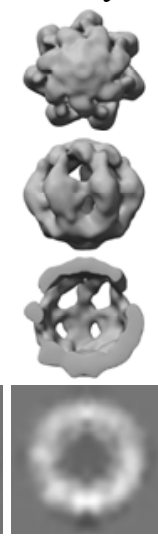

B

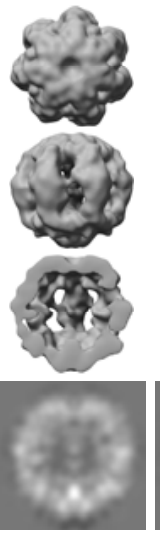

C

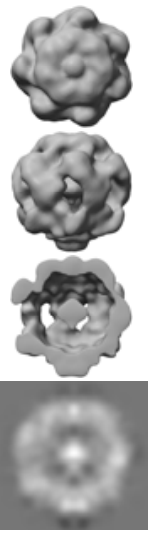

D

Figure 3. Structures of Four Conformations from Heterogeneous SR398- $\alpha \beta$-GroES-ATP Complex Data at about $20 \AA$ (A) Crystal-structure-like conformation. (B-D) Three representative expanded conformations. Some significant additional density exists inside the cavity of conformation D. 